\title{
Chemical Contribution to Surface-Enhanced Raman Scattering
}

\author{
B. N. J. Persson, ${ }^{1,2,3}$ Ke Zhao, ${ }^{4}$ and Zhenyu Zhang ${ }^{1,4}$ \\ ${ }^{1}$ Condensed Matter Sciences Division, Oak Ridge National Laboratory, Oak Ridge, Tennessee 37831, USA \\ ${ }^{2}$ Kavli Institute for Theoretical Physics, University of California, Santa Barbara, California 93106-4030, USA \\ ${ }^{3}$ IFF, FZ-Jülich, 52425 Jülich, Germany \\ ${ }^{4}$ Department of Physics and Astronomy, The University of Tennessee, Knoxville, Tennessee 37996-1200, USA
}

(Received 5 December 2005; published 22 May 2006)

\begin{abstract}
We present a new mechanism for the chemical contribution to surface-enhanced Raman scattering (SERS). The theory considers the modulation of the polarizability of a metal nanocluster or a flat metal surface by the vibrational motion of an adsorbed molecule. The modulated polarization of the substrate coupled with the incident light will contribute to the Raman scattering enhancement. We show that for a metal cluster and for a flat metal surface this new chemical contribution may enhance the Raman scattering intensity by a factor of $\sim 10^{2}$ and $\sim 10^{4}$, respectively. The new SERS process is determined by the electric field parallel to the surface of the metal substrate at the molecular binding site.
\end{abstract}

DOI: 10.1103/PhysRevLett.96.207401

PACS numbers: $78.30 .-\mathrm{j}, 78.68 .+\mathrm{m}$

About 30 years ago it was discovered that many molecules adsorbed on rough metal surfaces, in particular, silver surfaces, may exhibit Raman scattering cross sections a factor $\sim 10^{6}$ larger than that from the same molecules in the gas phase [1-3]. This so-called surface-enhanced Raman scattering (SERS) has gained new interest since the observation of giant SERS from single molecules located between closely spaced silver nanoclusters [4,5]. In a typical case the cluster diameter $\sim 10^{2} \mathrm{~nm}$ and the separation between the cluster surfaces at the "neck" $1-2 \mathrm{~nm}$. For such situations SERS enhancements up to $\sim 10^{14}$ have been reported $[4,5]$.

Numerous experiments and theories have shown that there are two different types of contributions to the SERS cross section, namely, electromagnetic field enhancement and chemical enhancement [6-10]. The chemical enhancement can be detected in experiments as a "first layer" effect. That is, the first monolayer of adsorbed molecules often exhibit a SERS cross section much larger than that from the second layer, in spite of the fact that the field enhancement should be nearly the same for both layers. In a typical situation for adsorbates on rough silver surfaces the enhancement from the chemical contribution is $\sim 10^{2}$ while the field enhancement is of order $\sim 10^{4}$. The field enhancement is derived from the fact that the electric field at the surface of a rough metal may be strongly enhanced, compared to the external "driving" field (such as that from a laser beam), when the frequency of the oscillating field $\omega$ is close to the resonant frequency of plasmon oscillations in the surface roughness protrusions. This is best illustrated by considering a metal nanocluster with radius $R$. If $R$ is much smaller than the wavelength of the external electromagnetic wave, then we can treat the cluster within the dipole approximation where the electric field on the surface of the cluster is of order $p / R^{3}$, with the induced dipole $p=\alpha_{p} E_{\text {ext }}$. For a free-electron-like metal, the polarizability $\alpha_{p}$ of the cluster is given by

$$
\alpha_{p}(\omega)=R^{3}\left[1-\frac{\omega}{\Omega}\left(\frac{\omega}{\Omega}+i \frac{\gamma}{\Omega}\right)\right]^{-1},
$$

where $\Omega=\omega_{\mathrm{p}} / \sqrt{ } 3$ is the resonant frequency of the plasma oscillations within the cluster $\left(\omega_{\mathrm{p}}\right.$ is the bulk plasma frequency of the metal) and $\gamma$ measures the effective damping (see below). Thus, the local electric field at a molecule adsorbed on the surface of the metal cluster is

$$
E_{\mathrm{loc}} \approx E_{\mathrm{ext}}\left[1-\frac{\omega}{\Omega}\left(\frac{\omega}{\Omega}+i \frac{\gamma}{\Omega}\right)\right]^{-1}=M(\omega) E_{\mathrm{ext}} .
$$

At resonance $\omega=\Omega,|M|=\Omega / \gamma$ and since typically the damping [11] $\gamma \approx 0.1 \Omega$ (or less), an enhancement of the electric field by a factor $\sim 10$ (or more) may occur. Since the Raman scattering cross section depends on the electric field as $\left|E_{\text {loc }}\right|^{4}$ [or, more accurately, as $\left|E_{\text {loc }}(\omega)\right|^{2} \mid E_{\text {loc }}(\omega-$ $\left.\omega_{0}\right)\left.\right|^{2}$, where $\omega \approx \Omega$ is the frequency of the incoming photon and $\omega_{0}$ the frequency of the adsorbate vibration involved in the Raman scattering process], a field enhancement of order $10^{4}$ (or more) is expected.

The chemical contribution to SERS has been attributed to charge transfer excitations between the metal and the adsorbates [12-15]. Briefly, the transfer of an electron from the metal to a molecular orbital on the adsorbate will change the potential in the molecule and induce a change in the position of the nuclei of the atoms in the molecule. When the electron tunnels (or jumps) back to the metal, the molecule will in general be left in a vibrationally excited state (a breakdown of adiabaticity). Such a charge transfer excitation has been estimated [12] to give a maximum enhancement of order $\sim 10^{2}$. Attempts at unified treatment of SERS have been presented in Ref. [16].

In this Letter, we propose a new mechanism for the chemical contribution to SERS from molecules adsorbed on a metal substrate (a nanocluster or a flat surface) by considering the modulation of the substrate polarizability due to the vibrational motion of the adsorbed molecules. 
We show that, when coupled with the incident light, the modulated polarization can contribute to a Raman scattering enhancement of the order $\sim 10^{2}$ for the case of silver nanoclusters, or of the order of $\sim 10^{4}$ for flat silver surfaces.

Consider first the Raman scattering from a gas phase molecule. The simplest treatment of the inelastic scattering process considers the dependence of the molecular (electronic) polarizability $\alpha_{\mathrm{m}}(Q)$ on the vibrational normal mode coordinate $Q$ involved in the Raman scattering. This treatment is accurate if the (laser) photon energy $\hbar \omega$ is not close to any molecular electronic excitation energies. The dipole moment induced in the molecule is

$$
p=\alpha_{\mathrm{m}}(Q) E_{\mathrm{ext}} \approx \alpha_{\mathrm{m}}(0) E_{\mathrm{ext}}+\alpha_{\mathrm{m}}^{\prime}(0) Q E_{\mathrm{ext}} .
$$

The first term in this expansion in $Q$ gives rise to elastically scattered light (Rayleigh scattering). The second term describes Raman scattering where the frequency of the radiation $\omega^{\prime}=\omega \pm \omega_{0}$, where $\omega$ and $\omega_{0}$ are the frequency of the incident photon and of the molecular vibration, respectively.

Consider next a molecule adsorbed on a small metal cluster. If the adsorbate would not interact chemically with the metal cluster, the Raman scattering from the moleculecluster complex can be obtained in the same way as for a gas phase molecule, except that $E_{\text {ext }}$ in (3) must be replaced by the local electric field $E_{\text {loc }}$ at the position of the molecule. Thus, in this case,

$$
p_{\text {Raman }}=\alpha_{\mathrm{m}}^{\prime}(0) Q E_{\mathrm{loc}}
$$

gives the Raman dipole.

When the molecule interacts chemically with the metal cluster, two new effects are possible, namely, (a) charge transfer excitations as described by earlier theories [1215] of the chemical contribution to SERS, and (b) a modulation of the cluster polarizability by the vibrational motion of the molecule as described by expanding the cluster polarizability $\alpha_{p}$ to linear order in $Q$. Process (a) is mainly caused by the perpendicular electric field at the surface of the metal cluster, while process (b) is determined by the parallel electric field, see Fig. 1. For a spherical metal cluster with a layer of adsorbed molecules, the polarizability of the cluster is given by (1) where the damping $\gamma$ has a contribution from electron scattering processes in the
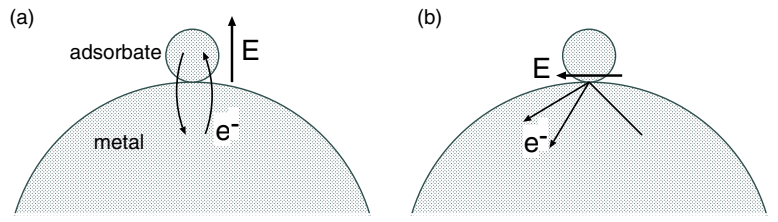

FIG. 1 (color online). Chemical contributions to SERS: (a) charge transfer excitations and (b) a modulation of the cluster polarizability by the vibrational motion of the molecule, involving only the parallel electric field at the molecule. bulk (as usually described by a Drude relaxation time $\tau_{\mathrm{D}}$ via $\left.\gamma_{0}=1 / \tau_{\mathrm{D}}\right)$ and a contribution from diffuselike scattering of the electrons from the surface of the cluster $[17,18]$. The latter contribution is proportional to the surface-to-volume ratio, i.e., to $1 / R$, and we write

$$
\gamma=\gamma_{0}+C \frac{v_{\mathrm{F}}}{R}
$$

where $v_{\mathrm{F}}$ is the Fermi velocity. For a silver cluster in vacuum, $C \approx 0.3$ as obtained theoretically [17] and also measured experimentally [11,19]. For a cluster with a monolayer of adsorbed atoms or molecules, the prefactor $C$ will be enhanced. Thus, for adsorbed $\mathrm{CO}$ and $\mathrm{C}_{2} \mathrm{H}_{4}$ one has $C \approx 1.0$ and $\approx 0.7$, respectively $[18,20]$. These molecules interact chemically with the silver cluster surface and give rise to large effective cross sections to scatter the silver metal electrons nonspecularly. On the other hand, very inert adsorbates such as the light noble gas atoms $\mathrm{Ne}$ and $\mathrm{Kr}$ do not interact chemically with silver and have very weak influence on the scattering of the metal electrons from the silver cluster surface, and are practically equivalent to vacuum $[18,20]$.

In Ref. [18] it has been shown that

$$
C=\frac{3}{8} n_{\mathrm{a}} \sigma_{\text {diff }}(\omega)
$$

where $n_{\mathrm{a}}$ is the number of adsorbed molecules per unit area, and $\sigma_{\text {diff }}(\omega)$ the effective cross section (at the excitation frequency $\omega$ ) for diffusive scattering of metal electrons from the adsorbed molecule. The function $\sigma_{\text {diff }}(\omega)$ depends on the nature of the chemical bond between the molecule and the substrate. For the systems which interest us here, there is an adsorbate induced resonant state centered at $\epsilon_{\mathrm{a}}$ a few electron volts above the Fermi surface and with the width $\Gamma$, and characterized by the local or projected density of states $\rho_{\mathrm{a}}=(\Gamma / 2 \pi)\left[\left(\epsilon-\epsilon_{\mathrm{a}}\right)^{2}+\right.$ $\left.(\Gamma / 2)^{2}\right]^{-1}$. For this case

$\sigma_{\text {diff }}=\frac{\sigma_{0} \pi \Gamma}{4 \epsilon_{\mathrm{F}} \hbar \omega} \int_{\epsilon_{\mathrm{F}}-\hbar \omega}^{\epsilon_{\mathrm{F}}} d \epsilon\left[\epsilon \rho_{\mathrm{a}}(\epsilon+\hbar \omega)+(\epsilon+\hbar \omega) \rho_{\mathrm{a}}(\epsilon)\right]$,

where $\sigma_{0}$ depends on the electron density of the metal substrate and on the symmetry of the resonant state [18]; for the $2 \pi^{*}$ state of $\mathrm{CO}$ on $\mathrm{Ag}, \sigma_{0} \approx 50 \AA^{2}$. In what follows we are only interested in $\omega \approx \Omega$ and we will not indicate the frequency dependence of $\sigma_{\text {diff }}$ explicitly. For $\mathrm{CO}$ and $\mathrm{C}_{2} \mathrm{H}_{4}, \sigma_{\text {diff }}$ is of order $\sim 5-10 \AA^{2}$. If we have only one molecule adsorbed on the cluster, then $n_{\mathrm{a}}=\left(4 \pi R^{2}\right)^{-1}$ and (6) becomes

$$
C=\frac{3}{32 \pi R^{2}} \sigma_{\text {diff }}
$$

The theory for the adsorbate contribution to $C$ is very similar to the theory used to calculate the influence of adsorbates on the surface resistivity of metals [21]. In 
fact, the main difference is that in the present case the cross section $\sigma_{\text {diff }}$ corresponds to optical frequencies (or excitation energies) $\hbar \omega \sim \hbar \Omega \sim 2-3 \mathrm{eV}$, while in surface resistivity applications only the zero frequency cross section enters. Nevertheless, the influence of different adsorbates on the cluster polarizability via the $C$ parameter is very similar to the influence the same adsorbates have on the surface resistivity. However, for molecules such as $\mathrm{CO}$ or $\mathrm{C}_{2} \mathrm{H}_{4}$ chemisorbed on silver $\sigma_{\text {diff }}(\Omega) \approx 10 \sigma_{\text {diff }}(0)$. This large enhancement comes from the fact that the relevant adsorbate induced resonant states involved in the electron scattering process are centered a few eV above the Fermi energy and hence can be nearly resonantly occupied at optical frequencies, while at very low frequencies only the tail of the resonant state at the Fermi energy can be involved in the scattering process.

For a metal cluster with a single adsorbed molecule, the cross section $\sigma_{\text {diff }}$, and hence the parameter $C$, depend on the chemisorption bond between the metal cluster and the adsorbate. The chemisorption bond will change when the nuclear positions of the atoms in the adsorbate change. Thus, $\sigma_{\text {diff }}$ (and $C$ ) can be viewed to depend parametrically on the vibrational normal mode $Q$ of the adsorbed molecule, leading to $\sigma_{\text {diff }}(Q) \approx \sigma_{\text {diff }}(0)+$ $\sigma_{\text {diff }}^{\prime}(0) Q$. Substituting this in (8) and using (5) and (1) gives, to leading order in $Q$,

$$
\alpha_{p}(\omega, Q) \approx M R^{3}+M^{2} \frac{3 i v_{\mathrm{F}}}{32 \pi \Omega} \sigma_{\mathrm{diff}}^{\prime}(0) Q,
$$

where $M$ is defined in Eq. (2). Thus, in this case

$$
p_{\text {Raman }}=i M(\omega) D \sigma_{\text {diff }}^{\prime}(0) Q E_{\mathrm{loc}},
$$

where $E_{\mathrm{loc}}=M E_{\mathrm{ext}}$ and $D=3 v_{\mathrm{F}} /(32 \pi \Omega)$. Since the interaction energy is of the form $\sim p E_{\text {ext }}$ we must remove the factor $M(\omega)$ in (10) when comparing it to direct SERS cross sections obtained using the Raman dipole (4). Thus, since the Raman intensity is proportional to the square of $p_{\text {Raman }}$, the ratio between the SERS cross section with and without the chemical contribution is

$$
\left[D \sigma_{\text {diff }}^{\prime}(0)\right]^{2}:\left[\alpha_{\mathrm{m}}^{\prime}(0)\right]^{2} \text {. }
$$

Assume that the adsorbed molecule has an electronic resonant state centered a few eV above the metal Fermi energy. The resonant state is derived from some molecular energy level $\epsilon_{\mathrm{a}}$, and we assume that the only dependence of $\sigma_{\text {diff }}$ on $Q$ comes from the variation of $\epsilon_{\mathrm{a}}(Q)$ with $Q$. For example, for $\mathrm{CO}$ chemisorbed on silver, the $2 \pi^{*}$ level in the gas phase molecule shifts below the vacuum energy upon adsorption, forming a $\Gamma \approx 1 \mathrm{eV}$ wide resonant state about 2-3 eV above the silver Fermi energy [22]. For the CO $2 \pi^{*}$ level, $\epsilon_{\mathrm{a}}^{\prime}(0) \approx-11 \mathrm{eV} / \AA$. For this case, under optimal conditions where the excitation energy $\hbar \Omega$ corresponds to roughly the separation between the metal Fermi energy and the center of the adsorbate induced resonant state, we get (see Ref. [18]) $\sigma_{\text {diff }}^{\prime}(0) \approx \sigma_{0} \epsilon_{\mathrm{a}}^{\prime} /(2 \hbar \Omega)$, where $\sigma_{0} \approx 50 \AA^{2}$ for silver. Thus, we expect $\sigma_{\text {diff }}^{\prime} \approx 10^{2} \AA$ for adsorbates such as $\mathrm{CO}$ and $\mathrm{C}_{2} \mathrm{H}_{4}$.

For typical SERS molecules such as $\mathrm{CO}$ or $\mathrm{C}_{2} \mathrm{H}_{4}$ one expects from dimensional arguments (and also observed in experiment) that $\alpha_{\mathrm{m}}^{\prime}(0) \approx 1 \AA^{2}$. For the surface plasmon excitation of a small silver cluster, $D=3 v_{\mathrm{F}} /(32 \pi \Omega) \approx$ $0.1 \AA$. Taking $\sigma_{\text {diff }}^{\prime} \approx 100 \AA$, the Raman cross section ratio $\left[D \sigma_{\text {diff }}^{\prime}(0)\right]^{2}:\left[\alpha_{\mathrm{m}}^{\prime}(0)\right]^{2}$ becomes $100: 1$, i.e., the SERS chemical enhancement factor is predicted to be of order $\sim 10^{2}$.

The SERS process considered above involves the electric field parallel to the surface of the metal substrate at the molecular binding site, and therefore exhibits a different surface selection rule than the standard charge transfer model of SERS. We note that in order to excite electronhole pairs in a metal, both energy and momentum must be supplied. Electromagnetic waves (photons) carry energy but very little momentum. Thus, to excite electron-hole pairs in a metal some "external" source of momentum is necessary. At metal surfaces, the surface breaks the translational invariance normal to the surface and a normal electric field can (and will) excite electron-hole pairs. However, a parallel electric field cannot excite electronhole pairs, at least not within the jellium model. But when adsorbed molecules occur on the surface they will break the translational invariance parallel to the surface and can supply it with the momentum necessary in order to excite electron-hole pairs. This opens up a new channel for energy dissipation which is closely related to the concept of surface resistivity. In the context of SERS (at resonance) where $\omega_{\mathbf{q}}=\omega_{\mathbf{q}^{\prime}}+\omega_{0}$, we have no electronic excitation in the final state but electronic excitations occur as intermediate states and these excitations must conserve the momentum. Thus, the breaking of the translational invariance parallel to the surface by the adsorbed molecules (and the resulting source of momentum) will open up a new SERS channel involving the parallel electric field.

The Raman scattering selection rule for adsorbates is most well defined when the substrate metal is flat $[9,23]$. The simplest treatment of the influence of adsorbed molecules on the optical properties of semi-infinite (free-electron-like) metals in the frequency region of the so-called anomalous skin effect, which interest us here, is the "slab model" developed in Ref. [24]. In this model the surface region of the metal is treated as a slab with the thickness $d=\delta$ given by the so-called skin depth $\delta=c / \omega_{\mathrm{p}}$, where $c$ is the light velocity and $\omega_{\mathrm{p}}$ the plasma frequency. The polarizability per unit surface area of this region is given by

$$
\chi=-\frac{\omega_{\mathrm{p}}^{2} d}{4 \pi \omega(\omega+i \gamma)} .
$$

The damping $\gamma$ has a contribution $\gamma_{1}$ derived from electron scattering from bulk defects, e.g., against phonons, and another contribution from electron scattering from the adsorbed molecules [21]: 


$$
\gamma=\gamma_{1}+\frac{3}{16} \frac{v_{\mathrm{F}}}{d} n_{a} \sigma_{\text {diff }} .
$$

Let us assume that (on average) there is one adsorbed molecule within the surface area $A_{0}$, so that $n_{\mathrm{a}} A_{0}=1$. The polarizability of the volume $d A_{0}$ is thus

$$
\alpha=\chi A_{0}=-\frac{\omega_{\mathrm{p}}^{2} d A_{0}}{4 \pi \omega(\omega+i \gamma)} .
$$

Expanding $\sigma_{\text {diff }}$ and $\alpha$ to linear order in $Q$, we get

$$
\alpha=-\frac{\omega_{\mathrm{p}}^{2} d A_{0}}{4 \pi \omega\left(\omega+i \gamma_{0}\right)}\left(1+\frac{3}{16} \frac{1}{i \omega} \frac{v_{\mathrm{F}}}{d} n_{\mathrm{a}} \sigma_{\text {diff }}^{\prime}(0) Q\right) .
$$

Thus, the Raman contribution to the fluctuating dipole $p=$ $\alpha E$ is

$$
p_{\text {Raman }}=\frac{i \omega_{\mathrm{p}}^{2}}{4 \pi \omega\left(\omega+i \gamma_{0}\right)} \frac{3}{16} \frac{v_{\mathrm{F}}}{\omega} \sigma_{\text {diff }}^{\prime}(0) Q E .
$$

Since for an $s$-polarized electromagnetic wave, the electric field is continuous at the surface (i.e., the same just inside the metal as just outside), the direct Raman scattering from the adsorbed molecule is determined by the fluctuating dipole $\alpha_{\mathrm{m}}^{\prime}(0) Q E$. Since the intensity of the Raman scattering is proportional to the square of the fluctuating dipole moment, assuming $\gamma_{0} \ll \omega$ we get the ratio

$$
\left[D(\omega) \sigma_{\text {diff }}^{\prime}(0)\right]^{2}:\left[\alpha_{\mathrm{m}}^{\prime}(0)\right]^{2},
$$

where

$$
D(\omega)=\left(\frac{\omega_{\mathrm{p}}}{\omega}\right)^{2} \frac{3}{64 \pi} \frac{v_{\mathrm{F}}}{\omega} .
$$

Using the plasma frequency (for silver) $\hbar \omega_{\mathrm{p}}=9 \mathrm{eV}$ gives for $\hbar \omega=2.5 \mathrm{eV}$ the chemical enhancement $\sim 6000$.

The model studied above predicts that the parallel electric field can also give rise to Raman scattering from adsorbate vibrations. Thus, if Raman scattering could be detected from, e.g., the $\mathrm{C}-\mathrm{O}$ stretch vibration (for $\mathrm{CO}$ adsorbed on a flat silver surface), using an $s$-polarized electromagnetic field, it would be a direct and stringent test of the theory presented in this Letter. Such an indirect excitation mechanism with an $s$-polarized field has its close analogy in IR measurements of dipole forbidden adsorbate vibrations [25-27] and surface resistivity $[21,28]$.

In summary, we have presented a new mechanism for the chemical contribution to SERS, where the SERS intensity is determined by the parallel electric field. We have shown that the modulation of the polarizability of a small metal cluster by the vibrational motion of an adsorbed molecule may enhance the Raman scattering from the adsorbate vibration by a factor of $\sim 10^{2}$. For adsorbates on a flat metal surface, the Raman scattering using the $s$-polarized field can be even more strongly enhanced (by a factor $\sim 10^{4}$ ), as compared to the direct coupling.
The contribution of B.P. to this work was performed while visiting ORNL and KITP. This work was supported in part by Oak Ridge National Laboratory, managed by UT-Battelle, LLC, for the U.S. Department of Energy under Contract No. DE-AC05-00OR22725, and by the National Science Foundation under Grants No. PHY9907949 and No. DMR-0306239.

[1] M. Fleischmann, P. J. Hendra, and A. McQuillan, Chem. Phys. Lett. 26, 163 (1974).

[2] D. L. Jeanmarie and R. P. V. Duyne, J. Electroanal. Chem. 84, 1 (1977).

[3] M. G. Albrecht and J. A. Creighton, J. Am. Chem. Soc. 99, 5215 (1977).

[4] H. Xu, E. J. Bjerneld, M. Kall, and L. Börjesson, Phys. Rev. Lett. 83, 4357 (1999).

[5] A. M. Michaels, M. Nirmal, and L. E. Brus, J. Am. Chem. Soc. 121, 9932 (1999); J. Jiang, K. Bosnick, M. Maillard, and L. Brus, J. Phys. Chem. 107, 9964 (2003).

[6] M. Moskovits, J. Raman Spectrosc. 36, 485 (2005).

[7] W. Akemann, A. Otto, and H. R. Schober, Phys. Rev. Lett. 79, 5050 (1997).

[8] Z. Kotler and A. Nitzan, J. Phys. Chem. 86, 2011 (1982).

[9] A. Campion and P. Kambhampati, Chemical Society Reviews 27, 241 (1998).

[10] P. Kambhampati, C. M. Child, and A. Campion, J. Chem. Soc., Faraday Trans. 92, 4775 (1996).

[11] B. Lamprecht, A. Leitner, and F. R. Aussenegg, Appl. Phys. B 68, 419 (1999).

[12] B. N. J. Persson, Chem. Phys. Lett. 82, 561 (1981).

[13] F. J. Adrian, J. Chem. Phys. 77, 5302 (1982).

[14] J. R. Lombardi, R. L. Birke, T. Lu, and J. Xu, J. Chem. Phys. 84, 4174 (1986).

[15] E. Heller, R. L. Sundberg, and D. Tannor, J. Phys. Chem. 86, 1822 (1982).

[16] H. Xu et al., Phys. Rev. Lett. 93, 243002 (2004); P. Johansson et al., Phys. Rev. B 72, 035427 (2005).

[17] E. Zaremba and B. N. J. Persson, Phys. Rev. B 35, 596 (1987).

[18] B. N. J. Persson, Surf. Sci. 281, 153 (1993).

[19] U. Kreibig and M. Vollmer, Optical Properties of Metal Clusters (Springer-Verlag, Berlin, 1995).

[20] K.-P. Charle, F. Frank, and W. Schulze, Ber. Bunsen. Phys. Chem. 88, 350 (1984); K.-P. Charle, W. Schulze, and B. Winter, Z. Phys. D 12, 471 (1989).

[21] B. N. J. Persson, J. Chem. Phys. 98, 1659 (1993).

[22] D. Schmeisser, J.E. Demuth, and Ph. Avouris, Chem. Phys. Lett. 87, 324 (1982); J. E. Demuth, D. Schmeisser, and Ph. Avouris, Phys. Rev. Lett. 47, 1166 (1981).

[23] P. Kambhampati, C. M. Child, M. C. Foster, and A. Campion, J. Chem. Phys. 108, 5013 (1998).

[24] B. N. J. Persson, Phys. Rev. B 44, 3277 (1991).

[25] Z. Y. Zhang and D. C. Langreth, Phys. Rev. B 39, 10028 (1989).

[26] C. J. Hirschmugl et al., Phys. Rev. Lett. 65, 480 (1990).

[27] B. N. J. Persson et al., Surf. Sci. 310, 314 (1994).

[28] A. I. Volokitin et al., Phys. Rev. B 52, 2899 (1995). 